\title{
Preface: DGAA 2nd Special Issue on Mean Field Games
}

\author{
Martino Bardi · Peter E. Caines • \\ Italo Capuzzo Dolcetta
}

Published online: 19 February 2014

C) Springer Science+Business Media New York 2014

This is the second special issue of Dynamic Games and Applications on Mean Field Games (MFG, in short). The first was published in December 2013 and collected original papers on various aspects of the MFG theory and applications. The two issues aim at giving a wide overview of the emerging trends in this fast growing research area. An earlier snapshot of the research on MFG can be found in the special issue of Networks and Hetereogeneous Media [1].

The theory of MFG is a branch of Dynamic Games which aims at modeling and analyzing complex decision processes involving a large number of indistinguishable rational agents who have individually a very small influence on the overall system and are, on the other hand, influenced by the mass of the other agents. The name comes from particle physics where it is common to consider interactions among particles as an external mean field which influences the particles themselves. In spite of the optimization made by rational agents, playing the role of particles in such models, appropriate mean field equations can be derived to replace the many particles interactions by a single problem with an appropriately chosen external mean field which takes into account the global behavior of the individuals. The introduction of a social component in the optimization criteria makes this theory so flexible that it can be applied to various fields and, for this reason, it is attracting an increasing interest from economists (micro and macro), engineers, biologist describing the animal behavior, and possibly sociologists and urban planners.

\footnotetext{
M. Bardi $(\bowtie)$

Dipartimento di Matematica, Università di Padova, via Trieste 63, 35121 Padova, Italy

e-mail: bardi@math.unipd.it

P. E. Caines

CIM and Department of Electrical \& Computer Engineering, McGill University, Montreal, QC H3A 2A7, Canada

e-mail: peterc@cim.mcgill.ca

I. Capuzzo Dolcetta

Dipartimento di Matematica, Sapienza Università di Roma, P.le A. Moro 2, 00185 Roma, Italy

e-mail: capuzzo@mat.uniroma1.it
} 
The theory originated in the independent work of J.-M. Lasry and P.-L. Lions [5-7], and of M.Y. Huang, P. E. Caines and R. Malhamé [2,4,3]. J.-M. Lasry and P.-L. Lions [5-7] started from the systems of $N$ elliptic partial differential equations (PDEs for short) that provide a Nash equilibrium in feedback form for $N$-person stochastic differential games, and found in the limit as $N \rightarrow \infty$ a system of two PDEs, where a classical Hamilton-JacobiBellman equation is coupled with a Kolmogorov-Fokker-Planck equation for the density of the players. They also observed that the MFG PDEs have strong links with other important fields of mathematics, such as quantum mechanics, fluid dynamics, optimal transportation, and optimal control of systems driven by PDEs. The more recent developments of the general mathematical theory lead to a PDE in infinite dimensions, called the Master Equation, whose analysis is a challenging open problem, see P.-L. Lions' courses on the site of the Collège de France http://www.college-de-france.fr/site/en-pierre-louis-lions/.

M.Y. Huang, P. E. Caines and R. Malhamé [2,3] first derived the MFG equations in the Linear-Quadratic-Gaussian case for large populations of uniform [2] and non-uniform agents [3] in models motivated by power control problems in cell phone communications and the methods of statistical mechanics. Then, in [4], M.Y. Huang, R. Malhamé, and P. E. Caines extended their results to nonlinear systems described by McKean-Vlasov SDEs for nonuniform populations of agents. They continued the development of the theory in a series of articles, also with other authors, demonstrating the existence of $\epsilon$-Nash equilibria for masses of agents using MFG PDE derived controls in a range of different settings, these included localized cost functions, social welfare maximization, consensus seeking systems, adaptive control systems and systems with major and minor agents.

The present issue contains five articles. The paper by Diogo Gomes and João Saúde is a survey of the MFG theory that the authors split into the classical approach and MFG in Master Equation form. It contains several recent contributions of Gomes' group and includes models of population dynamics and of financial markets.

The article by Fabio Bagagiolo and Dario Bauso treats dynamic demand management for electrical power systems supplying a large number of agents; within a MFG framework it is shown that a class of hysteretic thermostatic controls achieves network frequency stabilization while minimizing energy consumption.

Huibing Yin, Prashant G. Mehta, Sean P. Meyn and Uday V. Shanbag consider a class of noncooperative games arising from the synchronization of a large collection of controlled heterogeneous oscillators and via a non-linear eigenvalue analysis establish bounds on the efficiency loss between the mean-field equilibrium and the social welfare optimum.

The article by Vassili Kolokoltsov, Marianna Troeva, and Wei Yang, proves that the rate of convergence as the number of players $N$ tends to infinity in the mean-field approximation of controlled diffusions is of the order $1 / N$, using tools of the theory of nonlinear Markov processes.

Finally, the paper by Alessio Porretta contains the proof of existence of weak solutions for the stochastic MFG planning problem which amounts to driving the solution of a Kolmogorov-Fokker-Planck equation from its initial distribution to a given terminal one by a suitable choice of the terminal condition of the Hamilton-Jacobi-Bellman equation. His approach by energy methods allows notably to extend previous results by Lions beyond the case of purely quadratic Hamiltonians.

In conclusion, we thank Georges Zaccour, Editor-in-Chief of Dynamic Games and Applications, for proposing a special issue on MFG and for his support during the preparation of 
the two volumes, and all the contributors and the referees. We hope that the two special issues will contribute to the development of new activities in the field.

\section{References}

1. Camilli F, Capuzzo Dolcetta I, Falcone M (eds) (2012) Special issue on Mean Field Games. Netw Heterog Media 7(2):i-ii

2. Huang M, Caines PE, Malhamé RP (2003) Individual and mass behaviour in large population stochastic wireless power control problems: centralized and Nash equilibrium solutions. In: Proceedings IEEE Conference on Decision and Control, HI, USA, 98-103

3. Huang M, Caines PE, Malhamé RP (2007) Large-population cost-coupled LQG problems with nonuniform agents: individual-mass behavior and decentralized $\epsilon$-Nash equilibria. IEEE Trans Automat Control $52: 1560-1571$

4. Huang M, Malhamé RP, Caines PE (2006) Large population stochastic dynamic games: closed-loop McKean-Vlasov systems and the Nash certainty equivalence principle. Commun Inf Syst 6:221-251

5. Lasry J-M, Lions P-L (2006) Jeux à champ moyen. I. Le cas stationnaire. C R Acad Sci Paris 343:619-625

6. Lasry J-M, Lions P-L (2006) Jeux à champ moyen. II. Horizon fini et contrôle optimal. C R Acad Sci Paris 343:679-684

7. Lasry J-M, Lions P-L (2007) Mean field games. Jpn J Math 2:229-260 